\title{
Una nueva especie del género Bipinnula (Orchidaceae) para Chile
}

\section{A new species of genus Bipinnula (Orchidaceae) for Chile}

\author{
Pablo Bravo-Monasterio ${ }^{1,5^{*}}$, Gabriela Baeza-Horta 2,5 , Patricio Peñailillo ${ }^{3}$, Diego Alarcón 2,5 \& \\ DOMINGO CONTRERAS ${ }^{4}$
}

${ }^{1}$ Facultad de Ciencias Forestales, Universidad de Concepción, Laboratorio de Invasiones Biológicas (LIB), Casilla 160-C, Concepción, Chile.

${ }^{2}$ Departamento de Botánica, Facultad de Ciencias Naturales y Oceanográficas, Universidad de Concepción, Casilla 160-C, Concepción, Chile.

${ }^{3}$ Instituto de Biología Vegetal y Biotecnología, Universidad de Talca, 2 Norte 685, Casilla 747, Talca, Chile.

${ }^{4}$ Facultad de Ciencias Básicas, Universidad Católica del Maule, Avda. San Miguel 3605, Casilla 617, Talca, Chile.

${ }^{5}$ Instituto de Ecología y Biodiversidad (IEB), Facultad de Ciencias, Universidad de Chile, Casilla 653, Santiago, Chile.

*pbravom@udec.cl

\section{RESUMEN}

Se describe e ilustra una nueva especie, Bipinnula gabriel (Orchidaceae). Esta nueva especie habita en el tipo de bosque esclerófilo costero de Chile Central, en la localidad de Putú, Constitución, Región del Maule. Discutimos su identidad frente a dos taxones afines (Bipinnula plumosa var. plumosa y Bipinnula plumosa var. philipporum) y las diferenciamos en términos de sus nichos ecológicos. Los resultados señalaron que esta nueva especie posee notables diferencias morfológicas con las especies más próximas, además de diferencias ecológicas que apoyan a esta especie como nueva. Entregamos una descripción de la nueva especie y su hábitat junto a propuestas para su estado de conservación y claves para una determinación adecuada.

Palabras Clave: Taxonomía, Bipinnula, orquídea, nueva especie, Chile central.

\begin{abstract}
A new species Bipinnula gabriel (Orchidaceae) is described and illustrated. The new species inhabits the coastal sclerophyllous forest of Central Chile, in the locality of Putu, Constitución, Maule Region. We discussed its identity against two related taxa (Bipinnula plumosa var. plumosa and Bipinnula plumosa var. philipporum) and the differences in terms of their ecological niches. The results showed that the new species has remarkable morphological differences with closest species, in addition to ecological differences supporting this species as new. We described the new species and habitat along with approach for their conservation status and the key for proper determination.
\end{abstract}

KeYwords: Taxonomy, Bipinnula, orchid, new species, Central Chile.

\section{INTRODUCCIÓN}

En Chile, el género Bipinnula Comm. ex Juss. está representado por cuatro especies endémicas: Bipinnula fimbriata (Poepp.) I.M. Johnst., B. plumosa Lindl., B. taltalensis I.M. Johnst. y B. volckmannii Kraenzl. (Marticorena \& Quezada 1985, Lehnebach 2003, Gutiérrez 2005, Elórtegui \& Novoa 2009). En el año 1993, Van Nieuwenhuizen publica una nueva especie del género para Chile, Bipinnula apinnula Gosewijn, la cual es transferida al género Chloraea como Chloraea apinnula (Gosewijn) Szlach. (Szlachetko 2001) y más tarde a Ulantha como
Ulantha apinnula (Gosewijn) Szlach. (Szlachetko \& Tukałło 2008), lo cual no es apoyado por la filogenia basada en datos moleculares, donde aparece junto a Bipinnula volckmannii y a las restantes especies de Bipinnula estudiadas (Cisternas et al. 2012a).

Las especies chilenas del género Bipinnula se reconocen fácilmente por presentar los tépalos sepalinos laterales prolongados en una punta pectinado-pestañosa (Navas 1973, Lehnebach 1999), formada por apéndices filiformes recorridos de cilios. Estas características junto con el tamaño y forma del labelo son elementos de diagnóstico para el reconocimiento de las especies. 
En Chile, los representantes del género Bipinnula se distribuyen en la Cordillera de la Costa desde los $25^{\circ}$ latitud sur en la localidad de Paposo, hasta los $36^{\circ}$ de latitud sur en la localidad de Pelluhue (Gutiérrez 2005, Bravo-Monasterio \& Baeza-Horta obs. pers.) y por la Cordillera de los Andes desde $\operatorname{los} 31^{\circ}$ en la localidad de Corrales, Salamanca, hasta $36^{\circ}$ latitud sur en la localidad de Las Trancas, Chillán (Reiche 1910, Lehnebach 1999, Novoa et al. 2006).

Recientemente en el año 2010, en el marco de estudios de la flora de Putú, comuna de Constitución, Región del Maule, se recolectó un individuo del mencionado género cuya morfología era distinta a las especies descritas hasta ahora. Su identidad pareció corresponder a B. plumosa. Sin embargo, posteriores estudios sobre la base de material de herbario y literatura situaron a esta especie como una nueva orquídea para Chile.

\section{MATERIALES Y MÉTODOS}

Se recolectaron ejemplares para su posterior determinación, los cuales fueron comparados con las colecciones del género depositadas en los herbarios CONC y SGO.

Para determinar diferencias entre los nichos ecológicos de la nueva especie respecto a otras poblaciones de aquellas especies más afines morfológicamente, se realizó un análisis de componentes principales (PCA), donde se recopiló datos de localidades de Bipinnula plumosa var. plumosa, y $B$. plumosa var. philipporum (Kraenzl.) Reiche, que de acuerdo a la evidencia morfológica serían más afín a la especie nueva. Estas localidades se obtuvieron de los herbarios CONC, SGO y datos del herbario del Jardín Botánico Nacional (Patricio Novoa, com. pers.) y de Lucía Abello (com. pers.). A partir de estos datos se recopiló información ecológica que incluyó altitud e información bioclimática obtenidas para cada punto al sobreponerlas con capas de las siguientes variables bioclimáticas provenientes de Hijmans et al. (2005); el rango medio de temperatura o promedio de rangos de temperatura para cada período (bv02), índice de isotermalidad (bv03), estacionalidad de la temperatura expresada como desviación estándar de las temperaturas medias mensuales (bv04), temperatura máxima del mes más cálido (bv05), temperatura mínima del mes más frío (bv06), precipitación anual total (bv12), índice de estacionalidad de la precipitación expresada como desviación estándar de la precipitación mensual en relación con la precipitación media anual (bv15), precipitación del trimestre más cálido (bv18) y la precipitación del trimestre más frío (bv19). Los procesos de análisis geográfico hicieron uso del paquete de análisis raster de R (Hijmans \& Van Etten 2012, R Development Core Team 2012). El análisis de componentes principales se hizo mediante la herramienta de análisis princomp del paquete stats de R (R Development Core Team 2012).

\section{RESULTADOS}

La identidad de este nuevo taxón tiene algunas correspondencias morfológicas con B. plumosa var. plumosa, en la forma del labelo y con la variedad philipporum, en los márgenes fimbriados de la mitad apical del labelo. Sin embargo, existen diferencias en la forma de éste y en el tamaño de los sépalos laterales, junto con el tamaño de la prolongación filiforme y los cilios desplegados a ambos costados de dicha prolongación (Tabla I), características importantes en el estudio de las especies del género, cuyas descripciones están basadas en las estructuras florales con especial énfasis en el labelo y los sépalos.

Geográficamente tanto B. plumosa var. plumosa y $B$. plumosa var. philipporum se desarrollan en altitudes medias a altas asociadas principalmente a cordones montañosos, en cambio la nueva especie habita en la costa del Maule en elevaciones cercanas al nivel del mar (Fig. 3).

El análisis de componentes principales (PCA) diferencia dos componentes que dan cuenta del $86,16 \%$ de la varianza contenida en los datos empleados (componentes 1 con

TABLA I. Caracteres comparativos de B. gabriel con B. plumosa.

TABLE I. Comparing characters B. gabriel with B. plumosa

\begin{tabular}{|c|c|c|}
\hline CARACTERES & Bipinnula gabriel & Bipinnula plumosa \\
\hline Bráctea foliosa de la base del escapo & $3-3,5 \mathrm{~cm}$ & $2,5-2,8 \mathrm{~cm}$ \\
\hline Sépalo dorsal & Fuertemente lanceolado & Lanceolado \\
\hline Prolongación filiforme de los sépalos laterales & $1,0-1,3 \mathrm{~cm}$ de largo & $4,0-4,2 \mathrm{~cm}$ de largo \\
\hline Cilios de la prolongación filiforme & $0,3-0,5 \mathrm{~cm}$ de largo & $0,8-1,0 \mathrm{~cm}$ de largo \\
\hline Labelo & $\begin{array}{c}\text { Recorrido por } 5 \text { a } 7 \text { líneas verdes de la } \\
\text { base hasta el ápice }\end{array}$ & Sin presencia de líneas \\
\hline Color de los pétalos y sépalos & Verde limón & Verde oscuro \\
\hline
\end{tabular}


un $55,91 \%$ y 2 con $30,25 \%$ de la varianza). En la Tabla II se aprecia la correlación de las variables bioclimáticas consideradas en relación con los componentes principales. Las variables con mayor correlación con el componente 1 son la precipitación anual total (bv12), precipitación del trimestre más cálido (bv18), precipitación del trimestre más frío (bv19) y el índice de isotermalidad (bv03). Las variables que presentaron una mayor correlación con el componente 2 incluyen: rango medio de temperatura (bv02), altitud sobre el nivel del mar (alt), temperatura mínima del mes más frío (bv06) y temperatura máxima del mes más cálido (bv05). Al observar el gráfico del PCA (Fig. 4), se puede apreciar una mayor cercanía entre las características ecológicas de las poblaciones en las cuales se distribuye Bipinnula plumosa, incluyendo la variedad Bipinnula plumosa var. philipporum, la cual aparece como un extremo de un gradiente ecológico. En una ubicación separada del conjunto anteriormente mencionado, se aprecia a la especie nueva diferenciada en el eje del componente principal número 2 (Fig. 4). Lo anterior se interpreta como diferencias entre el nicho ecológico en su componente relacionado a las temperaturas y altitud sobre el nivel del mar, de la especie nueva respecto de las poblaciones de Bipinnula plumosa. Considerando el gradiente altitudinal, la población de la especie nueva se desarrolla a una altitud de $45 \mathrm{~m}$ s.n.m., mientras que las poblaciones de Bipinnula plumosa se desarrollan en un rango altitudinal entre los 650 y 1.200 m s.n.m. Desde el punto de vista de la ecología de estas especies, se aprecia una similitud o continuo entre las poblaciones de la especie típica Bipinnula plumosa y de la variedad Bipinnula plumosa var. philipporum, que se diferencian a su vez en los nichos ecológicos de la nueva especie.

\section{DESCRIPCIÓN DE LA ESPECIE}

Bipinnula gabriel P.A. Bravo et G.M. Baeza, sp. nov. TIPO: Chile, Región del Maule Prov. Talca. Comuna de Constitución, camino costero $3 \mathrm{~km}$ al norte de Putú, frente a pajonal de Cuchi, bajo matorral arborescente de litre
( $35^{\circ} 09^{\prime} \mathrm{S}, 72^{\circ} 14^{\prime} \mathrm{W}$ ), 45 m s.n.m, Bravo P. \& Baeza G. s/n 14-IX-2010 (Holotipo CONC 175892). (Fig. 1, 2A y 2B).

Inflorescence with more than 10 flowers, lateral sepals larger than $2.5 \mathrm{~cm}$ with threadlike prolongation up to 1.3 $\mathrm{cm}$ and margins provided with cilia up to $0.5 \mathrm{~cm}$, lip greater than $2.5 \mathrm{~cm}$.

Planta herbácea, en la base formando una pseudorroseta basal con hojas de 15-20×3-4 cm, anchamente lineares a angostamente lanceoladas, ápice agudo, márgenes enteros, semicarnosas, algo acanaladas en el nervio medio, verdes durante la antesis floral. Inflorescencia de hasta $60 \mathrm{~cm}$ de altura con 10-15 flores. Bráctea foliosa de $3-3,5 \times 0,5 \mathrm{~cm}$. Flores sésiles de color verde limón y blanco. Sépalo dorsal oblongo-lanceolado de 2,8-3,3×0,6 cm. Sépalos laterales fuertemente lanceolados de $2,5-2,7 \times 0,8 \mathrm{~cm}$, recorridos por 5 venas de color verde y provistos de una prolongación filiforme en el ápice del mismo de 1-1,3 cm de largo, la cual en sus márgenes lleva cilios delgados de 0,3-0,5 cm de largo. Pétalos lanceolados de 2,5-3×0,5-0,7 cm, de color verde claro y recorridos por 5 nervios verde oscuro. Labelo ovado a romboidal, entero de 2,5-3×1-1,5 cm, blanco, recorrido por 5 a 7 nervios de color verde claro provistos de papilas y crestas de color verde oscuro hacia la base, márgenes en su mitad apical con fimbrias pectinado-pestañosa verdosas de $0,3-0,4 \mathrm{~cm}$ de largo. Columna de $1 \times 0,3 \mathrm{~cm}$ alada, la cual es de color amarillo en la base y de color blanco en la parte media-apical con una línea naranja oblicua entre ambas, base de la columna con dos falsas fosas nectaríferas. Ovario oblongo-lanceolado erecto de $2,4 \times 0,7 \mathrm{~cm}$.

Floración: agosto a septiembre.

\section{ETIMOLOGía DEL EPÍTETO ESPECÍFICO}

Los dos primeros autores de este artículo dedican esta nueva especie a su hijo Gabriel Rodolfo Bravo Baeza. Que este descubrimiento lo llene de entusiasmo por explorar y conocer el mundo natural.

Clave dicotómica Para Reconocer a las especies del género Bipinnula Presentes en Chile.

1. Ápice del labelo con márgenes cubiertos de papilas

2. Sépalos laterales sin presencia de apéndices fimbriados

B. apinnula

2. Sépalos laterales con presencia de apéndices fimbriados

3. Planta de 50-60 cm alto, sépalos de 1,8-2,0 cm de largo, labelo recorrido por 7 líneas de dientes falcados

3. Planta de $80 \mathrm{~cm}$ de alto, sépalos de 2,5-3,0 $\mathrm{cm}$ de largo, labelo con pocos dientes falcados B. fimbriata

1. Ápice del labelo con márgenes pectinado-pestañoso.

4. Inflorescencia de 2 a 5 flores

4. Inflorescencia de 8 a 15 flores

5. Sépalos laterales de 2,5-2,7 cm de largo, prolongación filiforme que nace en el ápice del sépalo de 1,0-1,3 cm de largo, con ambos márgenes provistos de cilios finos de 0,3-0,5 cm de largo, labelo de 2,5-3,0 cm de largo.

B. gabriel

5. Sépalos laterales de 2,0-2,5 cm de largo, prolongación filiforme que nace en el ápice del sépalo de 4,0-4,2 cm de largo, con ambos márgenes provistos de cilios finos de $0,8-1,0 \mathrm{~cm}$, labelo de 1,3-2,2 $\mathrm{cm}$ de largo.

B. plumosa 


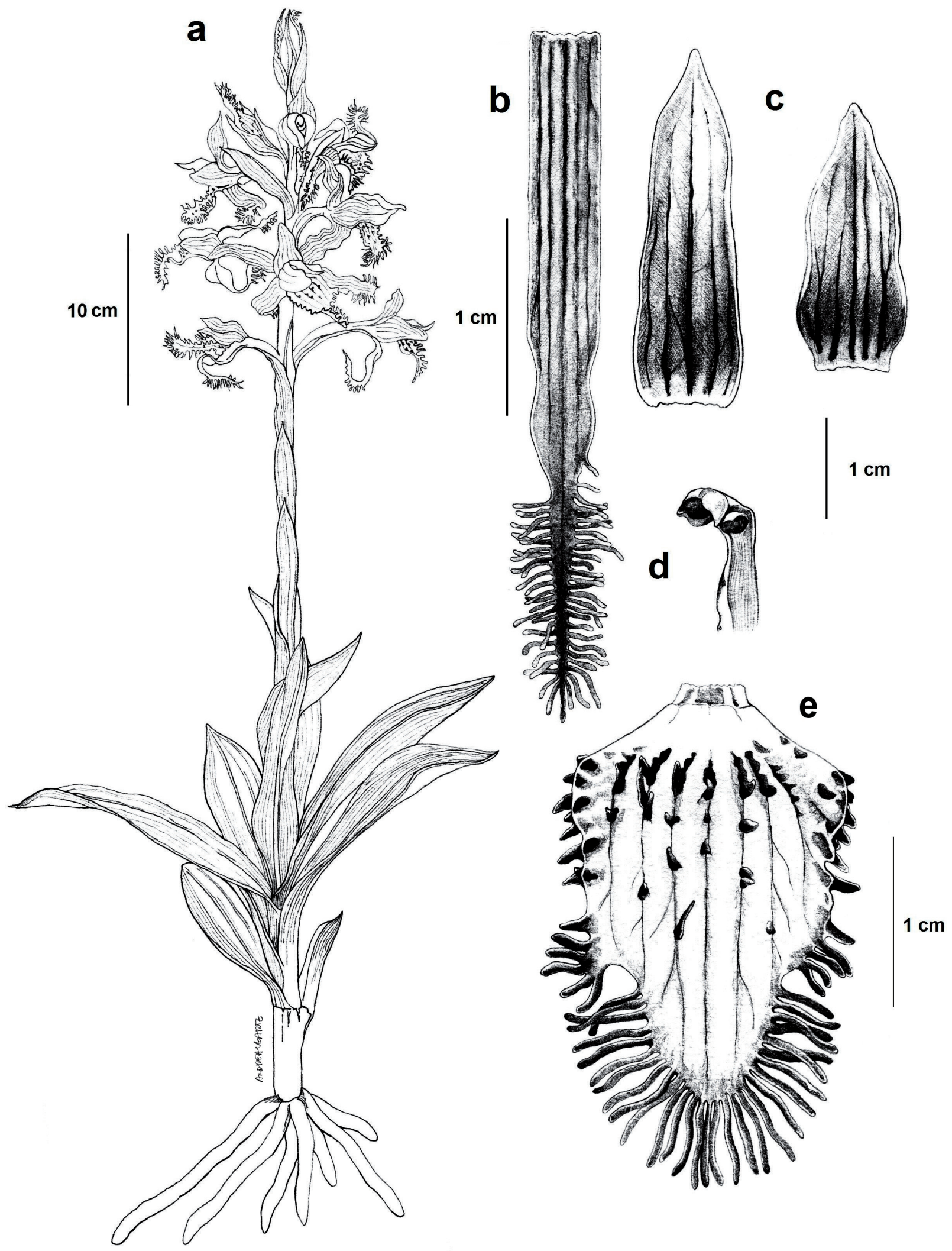

Figura 1. Bipinnula gabriel: a) Planta. b) Sépalo lateral. c) Sépalo dorsal y pétalo, d) Columna. e) Labelo. Dibujo original Andrea Ugarte. FIgURE 1. Bipinnula gabriel: a) Plant. b) Lateral sepal. c) Dorsal sepal and petal d) Column. e) Labellum. Original sketch Andrea Ugarte. 
DESCRIPCIÓN DEL HÁBITAT

Bippinula gabriel ha sido registrada sólo para la localidad tipo, cercana a Putú, comuna de Constitución, donde se observó una población de por lo menos 13 individuos, debido a que estimamos esta abundancia a partir de las visitas realizadas durante los años 2010, 2011, 2012 y 2013, asumiendo que los individuos no florecen en años consecutivos. Para el primer año encontramos 2 individuos con flores, 3 y 5 para los siguientes y 3 en el último año de observación. Notamos además que $B$. gabriel es simpátrica con Bipinnula fimbriata y Chloraea bletioides Lindl. Desde un punto de vista florístico crece en el matorral subarbustivo poco denso de Lithrea caustica (Molina) Hook. \& Arn. (Fig. 2C) con afloraciones rocosas y presencia de otras especies herbáceas, tales como Anemone decapetala Ard., Tristagma bivalve (Lindl.) Traub, Pasithea caerulea (Ruiz \& Pav.) D. Don entre otras. Vegetacionalmente la nueva especie se asocia a la comunidad de arbustos de Lithrea causticaAzara integrifolia perteneciente a la formación del "Bosque Caducifolio Maulino" descrita por Gajardo (1994), y al piso vegetacional "Bosque esclerófilo Mediterráneo costero de Lithrea caustica y Azara integrifolia" (Luebert \& Pliscoff 2006).

\section{DISCUSIÓN}

En Chile el número de especies de la familia Orchidaceae aún no se ha determinado de manera definitiva, ya que los estudios botánicos de la familia son escasos. Algunas aproximaciones sitúan el número de especies a 47 (Marticorena \& Quezada 1985) y en otras más recientes a 50, de las cuales 25 poseen un carácter endémico (Lehnebach 2003). Sin embargo, este último trabajo no consideró el hallazgo de $B$. apinnula realizado por Nieuwenhuizen en el año 1993. Recientemente otra especie ha sido descubierta en Chile y Argentina, correspondiente al género Gavilea, llamada G. gladysiae (Chemisquy 2009), este hallazgo más la incorporación de $B$. apinnula y $B$. gabriel, aumenta el número de taxa de especies de la familia Orchidaceae en Chile a 53 y de especies endémicas a 27.

En 1993, Van Nieuwenhuizen divide el género Bipinnula, sobre la base del número de flores, por inflorescencia y la morfología del labelo, en tres secciones: Bipinnula, Trilobatae y Multiflorae. La especie Bipinnula gabriel pertenece a la sección Multiflorae dado que presenta entre 10-15 flores; labelo entero, ancho, provisto de lamelas o escamas distantes; ápice de los sépalos laterales fimbriados, formando plumas con muchas lacinias largas; base del ginostemo con nectarios.

Sobre la base de las características de la sección Multiflorae, Szlachetko \& Margońska (2001) crean el género Jouyella Szlach., cuya especie tipo es J. fimbriata (Poepp.) Szlach. \& Marg., además de la especie tipo agrega J. philipporum (Kraenzl.) Szlach. \& Marg., J. plumosa (Lindl.) Szlach. \& Marg. y J. taltalensis (I.M.Johnst) Szlach. \& Marg., circunscribiendo el género Bipinnula a todas aquellas especies que presentan hojas marchitas durante

TABLA II. Correlaciones de las variables ecológicas estudiadas para las poblaciones de Bipinnula plumosa Lindl. var. plumosa, Bipinnula plumosa var. philipporum (Kraenzl.) Reiche y Bipinnula gabriel P.A. Bravo et G.M. Baeza, dentro del análisis de componentes principales.

TABLE II. Correlations of ecological variable for populations studied of Bipinnula plumosa Lindl. var. plumosa, Bipinnula plumosa var. philipporum (Kraenzl.) Reiche, y Bipinnula gabriel P.A. Bravo et G.M. Baeza, within the principal component analysis.

\begin{tabular}{lccc}
\hline VARiable Ecológica & Código & Componente 1 & Componente 2 \\
\hline Altitud sobre el nivel del mar & alt & 0,122 & $-0,475$ \\
Rango medio de temperatura & bv02 & & $-0,569$ \\
Índice de isotermalidad & bv03 & $-0,378$ & $-0,196$ \\
Estacionalidad de la temperatura & bv04 & 0,344 & $-0,310$ \\
Temperatura máxima del mes más cálido & bv05 & $-0,124$ & $-0,324$ \\
Temperatura mínima del mes más frío & bv06 & $-0,318$ & 0,355 \\
Precipitación anual & bv12 & 0,405 & 0,155 \\
Estacionalidad de la precipitación & bv15 & $-0,372$ & 0,214 \\
Precipitación del trimestre más cálido & bv18 & 0,395 & 0,385 \\
Precipitación del trimestre más frío & bv19 & \\
\hline
\end{tabular}



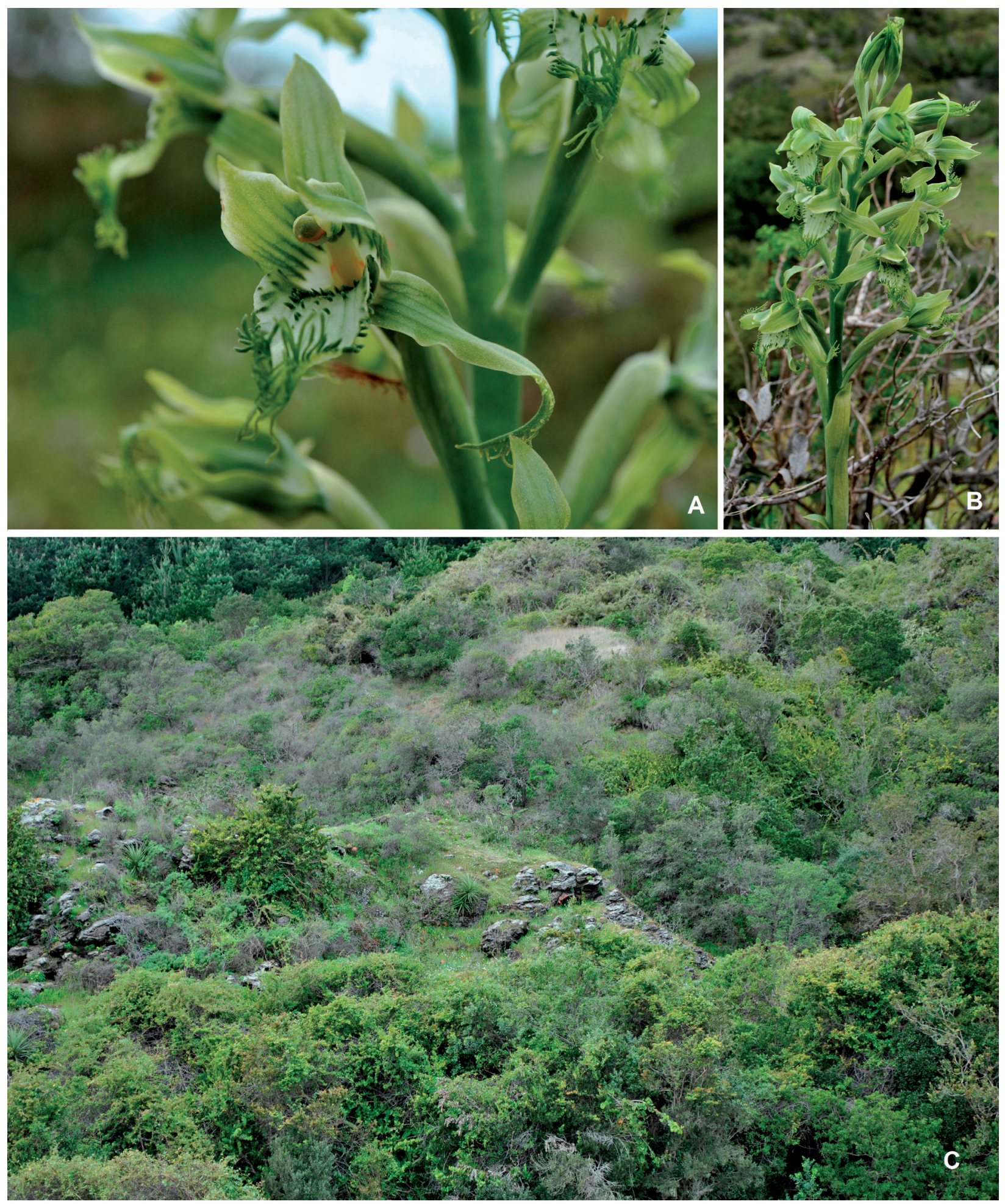

Figura 2. Bipinnula gabriel: A) Flor. B) Inflorescencia. C) Hábitat.

FIGURE 2. Bipinnula gabriel: A) Flower. B) Inflorescence. C) Habitat. 


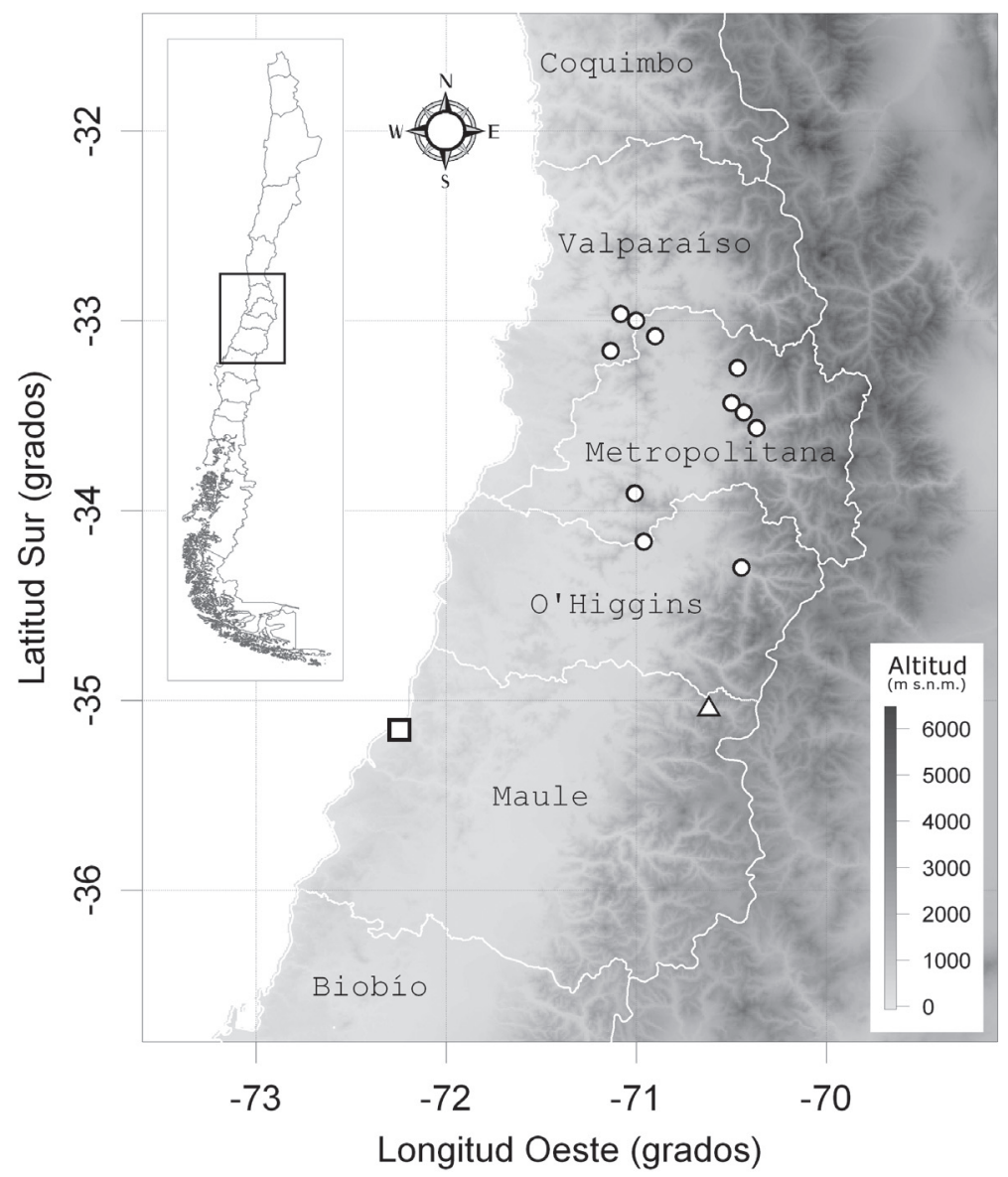

FiguRA 3. Mapa de ubicación geográfica y altitudinal de la poblaciones de Bipinnula gabriel (cuadrado), Bipinnula plumosa var. plumosa (círculos) y Bipinnula plumosa var. philipporum (triángulo).

FIgURE 3. Geographical and altitudinal map with localities of Bipinnula gabriel (square), Bipinnula plumosa var. plumosa (circles) y Bipinnula plumosa var. philipporum (triangle).

la floración, generalmente con una inflorescencia uniflora, flores horizontales en un pedicelo alargado más largo que el ovario, labelo siempre muy engrosado e insectiforme y tépalos relativamente pequeños. Un análisis filogenético de la subtribu Chloraeinae realizado por Cisternas et al. (2012a) muestra que los géneros Bipinnula y Gavilea Poepp. son parafiléticos y Chloraea Lindl. polifilético, resultados similares obtuvieron Chemisquy \& Morrone (2012). En el presente trabajo se utiliza un concepto genérico conservador tal como lo proponen los autores anteriores a la espera de una filogenia más consistente que abarque un mayor número de especies y géneros de la subtribu mencionada. Por otra parte, Cisternas et al. (2012b) sobre la base de sus estudios filogenéticos transfiere Geoblasta pennicillata (Rchb.f.) Hoehne ex Correa, al género Bipinnula, como $B$. pennicillata (Rchb.f.) Cisternas \& Salazar, de manera que el género Bipinnula quedaría monofilético y además aboga por la proposición de mantener las tres secciones en que fue dividido por Van Nieuwenhuizen (1993), uno de los escenarios señalados también por Chemisquy \& Morrone (2012).

Desde el punto de vista ecológico, las especies chilenas de la familia poseen muy pocos estudios (Bravo et al. 2012). Sin embargo, en este trabajo abordamos componentes ecológicos para observar las diferencias respecto del nicho ecológico de la especie morfológicamente más afín y la especie nueva. El PCA muestra que existen componentes del nicho que no comparten, donde se observa con mayor diferencia las variables asociadas a índices de temperaturas tanto altas como bajas, así como la participación diferenciada en el gradiente altitudinal, constituyentes del componente 2 del análisis. El hecho de tener un hábitat asociado a zonas muy cercanas a la costa, hace que las extremas térmicas sean atenuadas, lo que no ocurre en las zonas donde crece Bipinnula plumosa Lindl. En el plano imaginario que generan los componentes del PCA, se observa claramente en la Figura 4 que se encuentra separado de las distintas poblaciones descritas para las variedades de Bipinnula 
plumosa. Sobre la base de los antecedentes recogidos del punto de vista morfológico y ecológico, proponemos la sinonimia de la variedad philipporum con la especie B. plumosa por compartir el tamaño y forma del labelo, antecedente aclarado por Reiche (1910), y encontrarse dentro del mismo nicho ecológico.

Finalmente desde el punto de vista de la conservación, la población descrita en este trabajo se ubica en una zona de gran desarrollo vial e inmobiliario y colindante a plantaciones de Pinus radiata D. Don, las cuales se pueden considerar como amenazas para la sobrevivencia de la especie. Por lo tanto, se propone a $B$. gabriel en la clasificación de la UICN (2001) como Datos Insuficientes (DD), hasta conocer con seguridad si nos encontramos bajo un endemismo local. Si esto fuese así, B. gabriel se podría clasificar desde el punto de vista de los criterios B2ab (iii) en la categoría En Peligro (EN) debido a que sólo se conoce en su localidad tipo y se ha observado en la zona una disminución continua en la calidad de los hábitats nativos.

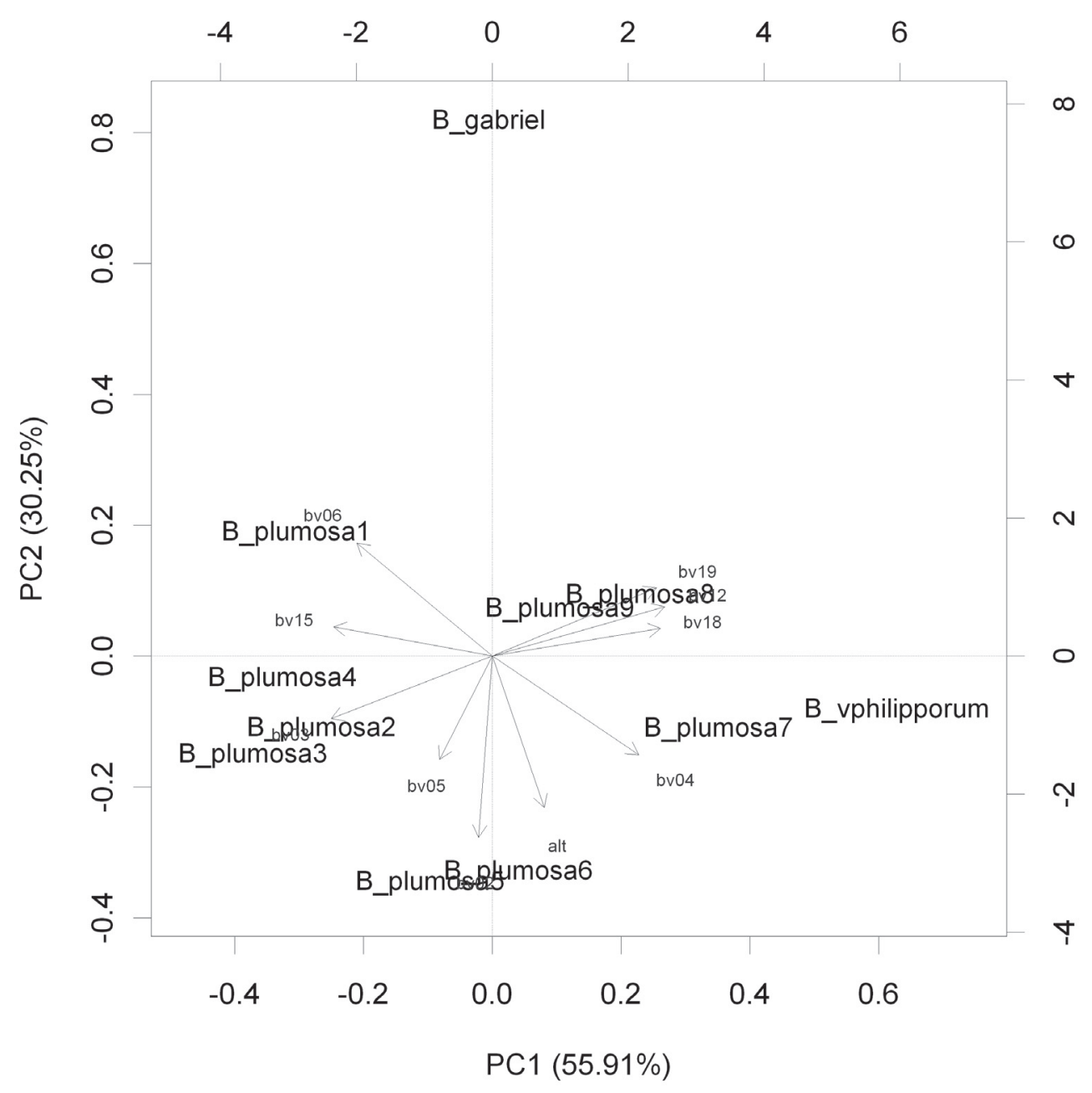

Figura 4. Ordenación PCA de la nueva especie y sus especies afines según características ecológicas de sus localidades. Bipinnula plumosa como B_plumosa, B. plumosa var. philipporum (Kraenzl.) Reiche, se expresa como B_vphilipporum y la nueva especie Bipinnula gabriel P.A. Bravo et G.M. Baeza, como B_gabriel. Las flechas en gris representan la magnitud de la correlación en los ejes o componentes principales, de acuerdo a la correlación de la Tabla II.

FiguRE 4. PCA ordenation of the new species and related species according to ecological data from their localities. Bipinnula plumosa as B_plumosa, B. plumosa var. philipporum (Kraenzl.) Reiche, as B_vphilipporum and the new species Bipinnula gabriel P.A. Bravo et G.M. Baeza, as B_gabriel. Gray arrows represent the magnitude of the correlation in the axis or components, according to the correlation of Table II. 


\section{CONCLUSIÓN}

Según las evidencias recogidas hasta la fecha, este taxón sería una especie endémica de la zona costera de la Región del Maule, debido a su restricción geográfica sólo a la población descrita en este trabajo. Sin embargo, uno de los problemas más claros con el grupo de las orquídeas es la falta de material depositado en los herbarios. Existe gran cantidad de aficionados al grupo de las orquídeas que conocen las plantas en el campo, pero no realizan colecciones de material de herbario, y en líneas generales, es posible que esta especie se encuentre en otras regiones o zonas litorales sin ser vista ni recolectada. Es necesario realizar nuevos viajes de recolección a localidades cercanas a la localidad tipo, o a localidades con ambientes similares, para dilucidar la distribución de la especie y afirmar con seguridad si encontramos un endemismo de la zona costera de la Región del Maule. Por otro lado, el eventual hallazgo de nuevas poblaciones de esta especie permitirá caracterizar mejor el nicho ecológico y proponer su estado de conservación con seguridad. No obstante, la información que se tiene hasta el momento y presentada en este trabajo, apoya la idea que se trata de una especie diferente en términos de nicho ecológico respecto de la especie más afín en términos morfológicos.

\section{AGRADECIMIENTOS}

Agradecemos a Andrea Ugarte por las ilustraciones de la nueva especie. Al Dr. Roberto Rodríguez, por sus valiosos comentarios al manuscrito. Alicia Marticorena y Gloria Rojas curadoras de los Herbarios CONC y SGO por facilitar nuestro trabajo consultando el material de herbario. Beca de Magíster del proyecto ICM 05-002 y al proyecto PFB-23. Diego Alarcón agradece a beca doctoral CONICYT.

\section{BIBLIOGRAFÍA}

Bravo-Monasterio, P., J. San Martín \& G. Baeza-Horta. 2012. Distribución, abundancia y fenología de orquídeas en un bosque caducifolio endémico de Chile Central. Polibotánica 33(1): 117-129.

Chemisquy, M.A. 2009. Gavilea gladysiae (Chloraeae: Orchidaceae), a new orchid from southern Argentina and Chile. Brittonia 61(3): 201-205.

Chemisquy, M.A. \& O. Morrone. 2012. Molecular phylogeny of Gavilea (Chloraeinae: Orchidaceae) using plastid and nuclear markers. Molecular Phylogenetics and Evolution 62: 889-897.

Cisternas, M.A., G.E. Salazar, G. Verdugo, P. Novoa, X. Calderón \& M.A. Negritto. 2012a. Phylogenetic analysis of Chloraeinae (Orchidaceae) based on plastid and nuclear DNA sequences. Botanical Journal of the Linnean Society
168(3): 258-277.

Cisternas, M.A., G.E. Salazar \& G. Verdugo. 2012b. Transfer of Geoblasta pennicillata to Bipinnula (Chloraeinae, Orchidaceae). Phytotaxa 64: 9-10.

Elórtegui, S. \& P. NovoA. 2009. Orquídeas de la Región de Valparaíso. Taller La Era. Viña del Mar. Chile. 82 pp.

Gajardo, R. 1994. La vegetación natural de Chile. Clasificación y distribución geográfica. Editorial Universitaria, Santiago Chile. $165 \mathrm{pp}$.

Gutiérrez, G. 2005. Hallazgo de Bipinnula taltalensis I.M. Johnston, en Paposo (II Región de Antofagasta). Chile. Chloris Chilensis Año $8 \mathrm{~N}^{\circ}$ 1. URL: http://www. chlorischile.cl.

HiJmans, R.J. \& J. van Etten. 2012. Raster: Geographic analysis and modeling with raster data. $\mathrm{R}$ package version 1.8-39. http://CRAN.R-project.org/package=raster.

Hijmans, R.J., S.E. Cameron, J.L. Parra, P.G. Jones \& A. Jarvis. 2005. Very high resolution interpolated climate surfaces for global land areas. International Journal of Climatology 25: 1965-1978.

Kraenzlin, F. 1904. Orchidacearum genera et species. Vol II, P. 1. Bayer \& Müller. Berlin. 1904. 143 pp.+ tafel.

Lehnebach, C.A. 1999. Current status of the Chilean orchid flora. Orchid Conservation News 2: 8-10.

Lehnebach, C.A. 2003. Preliminary checklist of the orchids of Chile. Botanical Journal of the Linnean Society 143: 449451.

Luebert, F. \& P. Pliscoff. 2006. Sinopsis bioclimática y vegetacional de Chile. Editorial Universitaria, 316 pp.

Marticorena, C. \& M. Quezada. 1985. Catálogo de la flora vascular de Chile. Gayana Botánica 42(1-2): 5-155.

Navas, L.E. 1973. Flora de la Cuenca de Santiago de Chile. Pteridophyta, Gimnospermae y Monocotyledoneae 1: 180181.

Nieuwenhuizen G.W.J. van 1993. Descubrimiento de una sorprendente orquídea en Talca. Gayana Botánica 50(1): 11-16.

Novoa, P., J. Espejo, M. Cisternas, M. Rubio \& E. Domínguez. 2006. Guía de Campo de las Orquídeas Chilenas. CORMA Ed. 120 pp.

R Development Core Team. 2012. R: A language and environment for statistical computing. R Foundation for Statistical Computing, Vienna, Austria. ISBN 3-900051-07-0, URL http://www.R-project.org/.

Reiche, K. 1910. Orchidaceae Chilenses. Ensayo de una Monografía de las Orquídeas de Chile. Anales del Museo Nacional de Chile, Sección Botánica, 18: i-ii, 1-88, 2 lám.

Szlachetko, D.L. 2001. Genera et species Orchidalium. 1. Polish Botanical Journal 46: 11-26.

SzlachetKo, D.L. \& H.B. MargońSKa. 2001. Genera et species Orchidalium. 4. Polish Botanical Journal 46: 123-125.

SzlachetKo, D.L. \& P. Tukalıo. 2008. Notes on the subtribe Chloraeinae (Orchidaceae). Acta Societatis Botanicorum Poloniae 77: 111-116.

UICN. 2001. Categorías y Criterios de la Lista Roja de la UICN: Versión 3.1. Comisión de Supervivencia de Especies de la UICN. UICN, Gland, Suiza y Cambridge, Reino Unido. ii $+33 \mathrm{pp}$.

Recibido: 17.07.12

Aceptado: 11.09.13 